\title{
CHAINS ON CR MANIFOLDS AND LORENTZ GEOMETRY
}

\author{
LISA K. KOCH
}

\begin{abstract}
We show that two nearby points of a strictly pseudoconvex CR manifold are joined by a chain. The proof uses techniques of Lorentzian geometry via a correspondence of Fefferman. The arguments also apply to more general systems of chain-like curves on CR manifolds.
\end{abstract}

0. Introduction. If $M$ is a nondegenerate CR manifold, its Fefferman metric is a conformal class of pseudo-Riemannian metrics on a circle bundle over $M$. The various CR invariants of $M$ may be described in terms of the conformal geometry of this metric; the description of chains in this setting is especially appealing. Recently, H. Jacobowitz showed that chains on a strictly pseudo-convex CR manifold connect pairs of nearby points. This paper presents a new proof of Jacobowitz's result which makes use of the Fefferman correspondence. We hope that this approach will yield new insights into the behavior of chains.

We begin with a definition of an abstract CR manifold of hypersurface type. We then sketch briefly a construction due to Lee [15] of the pseudo-Riemannian Fefferman manifold associated to a CR manifold, and discuss the special properties of such manifolds. Much of this discussion applies to a slightly more general class of pseudo-Riemannian manifolds.

We then proceed with our proof that chains on a strictly pseudoconvex CR manifold connect pairs of sufficiently nearby points. This proof applies also to the "pseudochains" associated to the more general pseudo-Riemannian manifolds discussed above. Finally, we discuss an example of a CR manifold with a system of pseudochains which are not chains.

I. CR manifolds and chains. An abstract almost CR manifold of hypersurface type is an odd-dimensional orientable manifold $M^{2 n+1}$ (which we shall always take to be smooth) together with a field of tangent hyperplanes $H^{2 n}$ on which a (smooth) complex structure $J, J: H \rightarrow H$ linear, $J^{2}=-\left.\mathrm{id}\right|_{H}$, is given. This $J$ is called a CR structure tensor on $M$. An almost CR manifold is called a CR manifold if its Nijenhuis torsion tensor,

$$
N(X, Y)=[J X, J Y]+J^{2}[X, Y]-J([X, J Y]+[J X, Y]),
$$

vanishes for $X, Y \in H$; a CR structure satisfying this condition is said to be formally integrable.

Let $\theta \in T^{*} M$ be a one-form which annihilates $H$. A choice of such a one-form is called a pseudo-Hermitian structure on $M$. The almost CR structure on $M$ is said to be nondegenerate if $\theta \wedge(d \theta)^{n} \neq 0$; this condition is independent of the choice of $\theta$.

Received by the editors April 28, 1987.

1980 Mathematics Subject Classification (1985 Revision). Primary 32F25; Secondary 53C50. 
There is another way of describing a CR structure to which we will need to refer later. Let $M^{2 n+1}$ be an odd-dimensional orientable smooth manifold. An almost CR structure on $M$ is an $n$-dimensional complex subbundle $T_{1,0}$ of the complexified tangent bundle CTM of $M$ such that $T_{1,0} \cap T_{0,1}=\{0\}$, where $T_{0,1}=$ $\overline{T_{1,0}}$. We set $H=\operatorname{Re}\left(T_{1,0} \oplus T_{0,1}\right) ; H$ is a real $2 n$-dimensional subbundle of $T M$. It carries a natural complex structure $J$ given by $J(V+\bar{V})=i(V-\bar{V})$ for $v \in T_{1,0}$. The integrability of the almost $\mathrm{CR}$ structure is equivalent in these terms to the requirement that $\left[T_{1,0}, T_{1,0}\right] \subset T_{1,0}$.

With respect to a choice of pseudo-Hermitian structure $\theta$ on $M$, the Levi form $L_{\theta}$ of $M$ is the Hermitian form on $T_{1,0}$ given by

$$
L_{\theta}(V, W)=i d \theta(V, \bar{W}) .
$$

The form $L_{\theta}$ depends on the choice of $\theta$, but it changes only by a conformal multiple if $\theta$ is changed. If $L_{\theta}$ is (positive) definite, $M$ is said to be strictly pseudoconvex. The Levi form $L_{\theta}$ gives rise to a real symmetric form on $H$ which we also call $L_{\theta}$.

It was first observed by Poincaré [21] in 1907 that there is no analog of the Riemann mapping theorem in several complex variables. That is, real hypersurfaces in $\mathbf{C}^{n}$ may be locally inequivalent under biholomorphic transformations. Poincaré conjectured that real hypersurfaces in $\mathbf{C}^{n}$ have certain biholomorphic invariants associated to them. The signature of the Levi form is one such invariant. Cartan [4] solved the equivalence problem for real hypersurfaces in $\mathbf{C}^{2}$; that is, he found a system of invariants on such hypersurfaces whose equality is necessary and sufficient for biholomorphic equivalence of the hypersurfaces.

Cartan also defined a biholomorphically invariant system of curves on hypersurfaces in $\mathbf{C}^{2}$, called chains. On $S^{3}$, or on the hyperquadric $H^{3}=\{(X, Y) \in$ $\left.\mathbf{C}^{2} \mid X-\bar{X}-i Y \bar{Y}=0\right\}$ (also called the Heisenberg group), the chains are the intersections of the hypersurface with affine complex lines. In general, chains are the solutions of a system of differential equations. To give this system explicitly for a three-dimensional CR manifold $M$, choose a pseudo-Hermitian structure $\theta$ on $M$, and let us define $\theta^{1}$ to be a complex one-form independent of $\theta$ which annihilates all vectors of the form $X+i J X$, for $X \in H$. For any complex-valued function $c$ on $M$, define $c_{0}, c_{1}$, and $c_{\overline{1}}$ by $d c=c_{0} \theta+c_{1} \theta^{1}+c_{\overline{1}} \overline{\theta^{1}}$. Then the chains on $M$ are the curves on $M$ which solve the equations

(1) $\theta^{1}=-\mu \theta$,

(2) $d \mu=\left(i \mu|\mu|^{2}+\frac{1}{2} i c \mu-\frac{1}{6} \bar{l}-\bar{b}|\mu|^{2}\right) \theta$,

where $b$ satisfies $d \theta=i \theta i \overline{\theta^{1}}+b \theta \wedge \overline{\theta^{1}}+\bar{b} \theta \wedge \overline{\theta^{1}}$, and $c$ and $l$ are given by $c=\overline{b_{1}}$ and $l=c_{1}-b c-2 i b_{0}$ (see [14]). Through any point of a hypersurface in $\mathbf{C}^{2}$, in any direction transverse to the holomorphic tangent plane $H$, there passes a unique chain. Chains carry a distinguished parameter which is defined up to a real projective transformation.

The work of Cartan was extended by Chern and Moser [6] to arbitrary abstract CR manifolds of hypersurface type. They also discuss a normal form for the equations defining a CR manifold $M$, produced by osculating $M$ by an image of the hyperquadric. This osculation takes place at a point $p$ along a chain passing through $p$.

Another way of describing chains is due to Fefferman [8]. For a strictly pseudoconvex hypersurface $M^{2 n+1} \subset \mathrm{C}^{n+1}$, he constructs a Lorentz metric on $M \times S^{1}$, 
defined up to a restricted conformal multiple; the conformal factor is required to be a function constant on the $S^{1}$ factor. The fibers $S^{1}$ are null geodesics of the metrics in this conformal class; the other null geodesics project to the chains of $M$.

Burns and Shnider [2] and Burns, Diederich, and Shnider [3] give an intrinsic construction of the Fefferman metric, thereby generalizing the correspondence to apply to any nondegenerate CR manifold of hypersurface type. For a CR manifold $M$ of Levi signature $(p, q)$, they obtain a pseudo-Riemannian manifold $L$ of signature $(2 p+1,2 q+1)$ on a certain circle bundle over $M$. If $M$ is strictly pseudoconvex, this pseudo-Riemannian manifold is of Lorentz signature. Burns, Shnider, and Diederich's construction involves embedding Chern's CR structure bundle for $M$ in the conformal structure bundle of $L$. In this way, they show that all CR invariants of $M$ may be recovered from the (restricted) conformal geometry of $L$.

II. Construction and characterization of the Fefferman metric. Lee [15] has given a more-direct construction of the Fefferman metric of an abstract CR manifold, which we shall review here. For a nondegenerate CR manifold $M^{2 n+1}$ of Levi signature $(p, q)$, the Fefferman metric is a canonically defined pseudo-Riemannian metric of signature $(2 p+1,2 q+1)$, determined up to a restricted conformal factor, on a certain circle bundle over $M$. This underlying circle bundle is the quotient $C=B^{*} / \mathbf{R}^{+}$of the canonical bundle $B$ of $M$ with its zero section removed, by the natural action of $\mathbf{R}^{+}$. Lee's construction of the Fefferman metric on $C$ gives a particular representative of the conformal class in terms of a choice of pseudoHermitian structure on $M$. Lee shows that the restricted conformal class of this metric is invariant under change of pseudo-Hermitian structure on $M$.

Choose a pseudo-Hermitian structure on $M$; that is, choose a (real) one-form $\theta$ on $M$ annihilating the hyperplane field $H$. Let $T$ be the unique vector field on $M$ so that $\theta(T)=1$ and $T\rfloor d \theta=0$. Choose $n$ complex one-forms on $M$ so that the $\theta^{\alpha}$, restricted to $T_{1,0}$, form a basis of $T^{1,0}$, and so that $\theta^{\alpha}(T)=0$.

These choices determine a (local) section $\varsigma_{0}=\theta \wedge \theta^{1} \wedge \cdots \theta^{n}$ of $K^{*}$, and thus also a section of $C$. Let $\gamma$ be the variable $(\bmod 2 \pi)$ on the fibers of $C$ such that $\left[e^{i \gamma} \zeta_{0}\right]=[\zeta]$, where $[\zeta]$ is an equivalence class of $(n+1,0)$-forms at a point of $M$. Let $K$ be the vertical (i.e., tangent to the fibers) vector field such that $d \gamma(K)=1$.

For a CR manifold $M$ of Levi signature $(p, q)$, the Fefferman metric on $C$ will be the pseudo-Riemannian metric $d s^{2}=L_{\theta}+2 \theta \sigma$ of signature $(2 p+1,2 q+1)$, where $\sigma$ is a canonically specified real one-form on $C$ with $\sigma(K) \neq 0$. To this end, let $\eta$ be an $n$-form on $C$ satisfying $\varsigma=\theta \wedge \eta, \tilde{T}\rfloor \eta=0$ for any lift $\tilde{T}$ of $T$ to $C$. Lee shows that $\eta$ is unique. The one-form $\sigma$ is then chosen to satisfy

(1) $d \varsigma=i(n-2) \sigma \wedge \varsigma$,

(2) $\sigma \wedge d \eta \wedge \bar{\eta}=\operatorname{Tr}(d \sigma) i \sigma \wedge \theta \wedge \eta \wedge \bar{\eta}$.

Lee shows that conditions (1) and (2) determine $\sigma$ uniquely, and that the resulting metric $d s^{2}=L_{\theta}+2 \theta \sigma$ agrees with the one defined by Fefferman on nondegenerate CR hypersurfaces in $\mathbf{C}^{n+1}$.

Sparling has shown (see $[\mathbf{2 2}, \mathbf{1 0}]$ ) that a pseudo-Riemannian manifold $L^{2 n+2}$ of signature $(2 p+1,2 q+1)$ is locally the Fefferman manifold of some nondegenerate CR manifold $M^{2 n+1}$ of Levi signature $(p, q)$ if and only if $L$ admits a null Killing 
vector field $K$ satisfying

(1) $K\rfloor C=0, K\rfloor \mathrm{Sch}=0$,

(2) $\operatorname{Ric}(K, K)>0$,

where $C$ is the Weyl tensor of $L$, Sch is the Schouten tensor, and Ric is the Ricci tensor. These conditions are invariant under conformal changes of metric on $L$ for which $K$ is a Killing field of both the new and old metrics. In the presence of condition (1), $\operatorname{Ric}(K, K)$ is actually constant [10]. If $L$ is of Lorentz signature, then for any null Killing field $K$ we have $\operatorname{Ric}(K, K) \geq 0$, and the significance of the strict inequality in the second condition is to make $\operatorname{rot}(K)$ nondegenerate, where

$$
\operatorname{rot}(K)(X, Y)=\frac{1}{2}\left\{\left\langle\nabla_{X} K, Y\right\rangle-\left\langle\nabla_{Y} K, X\right\rangle\right\} \quad \text { for } X, Y \perp K .
$$

This follows from an examination of Raychaudhuri's equation for null geodesics [12] (note that a null Killing field is geodesic, shearfree, and divergence free), or from some calculations we shall make in the next section. Observe that $\operatorname{rot}(K)(X, K)=0$ for any $X \in K^{\perp}$, and that $K^{\perp}$ has odd dimension $2 n+1$. Since $K$ is a null Killing field, $\operatorname{rot}(K)(X, Y)=\left\langle\nabla_{X} K, Y\right\rangle$. Thus $\operatorname{rot}(K)$ is nondegenerate if and only if, for each $X \in K^{\perp}, X \notin \operatorname{span}(K)$, there is a $Y \in K^{\perp}, Y \notin \operatorname{span}(K)$, such that $\left\langle\nabla_{X} K, Y\right\rangle \neq 0$. This means that $\nabla K$ is a nondegenerate linear transformation from $K^{\perp} / K$ to $K^{\perp} / K$. We show later in a more general context that $(\nabla K)^{2}=$ $-\left.\operatorname{Ric}(K, K) \cdot \operatorname{id}\right|_{K^{\perp} / K}$, and thus that $\nabla K$ projects to (a multiple of) a CR structure tensor on $M$.

III. Lorentz metrics and pseudochains. More generally, let $L^{2 n+2}$ be a real line bundle or circle bundle (with Lorentz metric $\langle$,$\rangle ) over an odd-dimensional$ orientable manifold $M^{2 n+1}$, such that the vertical null vector field $K$ is a null Killing field satisfying the conditions

(1) $\operatorname{rot}(K)$ is nondegenerate,

(2) $\langle C(X, K) K, X\rangle=0$ for $X \in K^{\perp}$,

where $C$ is the Weyl conformal curvature tensor of $L$. We shall see that the restricted conformal class of $(L,\langle\rangle$,$) determines an almost CR structure on M$, together with a system of curves (given by projecting the null geodesics of $L$ onto $M)$ which we shall call pseudochains.

The holomorphic tangent plane field $H^{2 n}$ is given, as before, by projecting $K^{\perp}$ onto $M$. In general, however, the CR structure tensor $J$ will not be simply the projection of $\nabla K$. To define $J$, we must recall the definitions of null sectional curvature and make a few observations about null sectional curvatures of vertical null planes.

DEFINITION [11]. Let $N$ be a null tangent vector at a point $p$ to a Lorentz manifold $L$; let $X$ be any spacelike vector perpendicular to $N$ at $p$. (Then $N \wedge X$ is a null plane containing $N$.) The null sectional curvature $\kappa_{N}(N \wedge X)$ of the null plane $N \wedge X$ with respect to $N$ is

$$
\kappa_{N}(N \wedge X)=\langle R(X, N) N, X\rangle /\langle X, X\rangle .
$$

This curvature is independent of the choice of $X$ in $N \wedge X$, but it depends quadratically on the choice of $N$ in $N \wedge X$. 
The Weyl conformal curvature tensor of an $m$-dimensional pseudo-Riemannian manifold is defined by

$$
\begin{aligned}
\langle C(X, Y) Z, W\rangle= & \langle R(X, Y) Z, W\rangle \\
& +\frac{1}{m-2}\{\langle X, W\rangle \operatorname{Ric}(Z, Y)-\langle X, Z\rangle \operatorname{Ric}(W, Y) \\
& +\langle Y, Z\rangle \operatorname{Ric}(W, X)-\langle Y, W\rangle \operatorname{Ric}(Z, X)\} \\
& +\frac{1}{(m-1)(m-2)} R(\langle X, Z\rangle\langle Y, W\rangle-\langle X, W\rangle\langle Y, Z\rangle) .
\end{aligned}
$$

In particular, for $X, Y \perp K$ we have

$$
\langle C(X, K) K, Y\rangle=\langle R(X, K) K, Y\rangle-\frac{1}{2 n} \operatorname{Ric}(K, K)\langle X, Y\rangle .
$$

Therefore, if $\langle C(X, K) K, X\rangle=0$ for all $X \perp K$, we see that

$$
\kappa_{K}(K \wedge X)=\frac{1}{2 n} \operatorname{Ric}(K, K)
$$

Thus the sectional curvatures of all vertical null planes are equal; let $\kappa_{K}$ denote their common value. To show $\kappa_{K}$ is positive, write

$$
\langle R(X, K) K, X\rangle=\left\langle\nabla_{X} \nabla_{K} K, X\right\rangle-\left\langle\nabla_{K} \nabla_{X} K, X\right\rangle-\left\langle\nabla_{[X, K]} K, X\right\rangle .
$$

Assume that $X$ is extended so that $[X, K]=0$. Then

$$
\begin{aligned}
\langle R(X, K) K, X\rangle & \left.=-\left\langle\nabla_{K} \nabla_{X} K, X\right\rangle \quad \text { (using } \nabla_{K} K=0\right) \\
& =-K\left\langle\nabla_{X} K, X\right\rangle+\left\langle\nabla_{X} K, \nabla_{K} X\right\rangle .
\end{aligned}
$$

But $\left\langle\nabla_{X} K, X\right\rangle=0$ since $K$ is Killing, and $[X, K]=0$, so

$$
\langle R(X, K) K, X\rangle=\left\langle\nabla_{X} K, \nabla_{X} K\right\rangle .
$$

In terms of null sectional curvature,

$$
\kappa_{K}\langle X, X\rangle=\left\langle\nabla_{X} K, \nabla_{X} K\right\rangle \text {. }
$$

Now $\nabla_{X} K \in K^{\perp}$, since $\left\langle\nabla_{X} K, K\right\rangle=\frac{1}{2} X\langle K, K\rangle=0$. And $\nabla_{X} K$ is not a multiple of $K$, since the assumption that $\operatorname{rot}(K)$ is nondegenerate means that $\left\langle\nabla_{X} K, Y\right\rangle \neq 0$ for some $Y \in K^{\perp}$. Therefore, $\nabla_{X} K$ is a nonzero spacelike vector, as is $X$, and

$$
\kappa_{K}=\frac{\langle R(X, K) K, X\rangle}{\langle X, X\rangle}=\frac{\left\langle\nabla_{X} K, \nabla_{X} K\right\rangle}{\langle X, X\rangle}>0 .
$$

We are now ready to define the CR structure tensor on $M$.

PROPOSITION. $J=\pi\left(\left.\left(1 / \sqrt{\kappa_{K}}\right) \nabla K\right|_{K^{\perp}}\right)$ is a CR structure tensor on $M$.

ProOF. We must show that $J^{2}(X)=-X$ for all $X \in \pi\left(K^{\perp}\right)$; that is, we must show that $(\nabla K)^{2}(X)=-\kappa_{K} \cdot X(\bmod K)$ for all $X \in K^{\perp}$. It suffices to show that $\left\langle(\nabla K)^{2}(X), Y\right\rangle=-\kappa_{K}\langle X, Y\rangle$ for all $X, Y$ in $K^{\perp}$.

$$
\begin{aligned}
\left\langle(\nabla K)^{2}(X), Y\right\rangle & =\left\langle\nabla_{\nabla_{X} K} K, Y\right\rangle=-\left\langle\nabla_{Y} K, \nabla_{X} K\right\rangle \quad \text { (since } K \text { is Killing) } \\
& =-X\left\langle\nabla_{Y} K, K\right\rangle+\left\langle\nabla_{X} \nabla_{Y} K, K\right\rangle \\
& =0+\langle R(Y, K) X, K\rangle=-\langle R(Y, K) K, X\rangle \\
& \left.=-\frac{1}{2 n} \operatorname{Ric}(K, K)\langle X, Y\rangle \quad \text { (since }\langle C(X, K) K, Y\rangle=0\right) \\
& =-\kappa_{K}\langle X, Y\rangle .
\end{aligned}
$$


Here we have used the identity $\nabla_{A} \nabla_{B} K-\nabla_{\nabla_{A} B} K=R(A, K) B$, which holds for any Killing field $K$ and any vectors $A$ and $B$, in the fourth equality; we have also assumed during the calculation that $[X, Y]=0$.

The CR structure tensor we have just defined may or may not be integrable. Graham [10] shows that the curvature conditions $K\rfloor C=0, K\rfloor K\rfloor$ Sch $=0$ are sufficient to ensure integrability (where $C$ is the Weyl tensor and Sch is the Schouten tensor of $L$ ). Of course, every CR structure tensor on a 3-dimensional manifold is integrable. If $\kappa_{K}$ is constant, then $K$ may be replaced by the new Killing field $K_{1}=K / \sqrt{\kappa_{K}}$, and then the CR structure tensor becomes just $\pi\left(\left.\nabla K_{1}\right|_{K_{1}^{\perp}}\right)$.

Since the flow of $L$ along $K$ is isometric, it takes null geodesics of $L$ to null geodesics of $L$. This ensures that, by projecting null geodesics of $L$ to $M$, we obtain a system of curves on $M$ determined by initial point and initial tangent direction. This initial direction must be transverse to $H$, for curves tangent to $H$ cannot be the projection of null geodesics of $L$.

These curves are just the chains of $M$ in the CR structure induced from $L$, if $L$ satisfies Sparling's conditions; if Sparling's conditions are not satisfied, then they will generally differ from the chains. We shall call curves of such systems pseudochains. It is the object of the next section to show that pseudochains connect nearby points of strictly pseudoconvex CR manifolds. This includes the result of Jacobowitz [14] that chains connect nearby points of a strictly pseudoconvex CR manifold, as the special case where the pseudochains are derived from a Lorentz manifold which is locally Fefferman.

So far, we have been considering Lorentz manifolds $L$ which are line bundles or circle bundles over the base $M$. However, if the fibers of $L$ are circles, we may replace $L$ with a line bundle $\tilde{L}$ over $M$ (or, at least, over an open neighborhood $U$ of $M$ ), so that $\tilde{L}$ is a covering space of $L$. That is, we may "unroll" the circular fibers into lines. To avoid confusion, in the next section we assume that all our Lorentz manifolds are line bundles over $M$. Since the theorem we wish to prove is of a local nature, we may make this assumption without loss of generality.

\section{Connectivity theorem and lemmas.}

THEOREM. Let $M^{2 n+1}$ be a strictly pseudoconvex CR manifold, with pseudochain structure derived from the conformal class of the Lorentz manifold $L^{2 n+2}$. Then, for any point $p \in M$ and any neighborhood $U$ of $p$, there is a neighborhood $V$ of $p, V \subset U$, so that $p$ can be joined to any distinct point $q \in V$ by a smooth pseudochain remaining in $V$.

The idea of the proof is to show that the lightcone of a point $\hat{p}$ of $L$, where $\pi(p)=\hat{p}$, is shaped so that the projection map $\pi: L \rightarrow M$ takes an appropriate portion of it onto a neighborhood of $p$. Since the lightcone of $\hat{p}$ consists of those points of $L$ which can be reached from $\hat{p}$ by a smooth null geodesic, this is equivalent to showing that $p$ can be joined to every point of the neighborhood by a pseudochain.

We begin by recalling that null sectional curvatures of all vertical null planes along a given fiber $\gamma=\pi^{-1}(p)$ are equal to the same positive constant. From Jacobi's equation, a point $\hat{p} \in \gamma$ must then have future and past null focal points along $\gamma$ of order $2 n$. Now choose a vertical hypersurface $\Gamma$ tangent to the lightcone of $\hat{p}$ along $\gamma$; Lemma $\mathrm{P}$ enables us to show that every fiber near $\gamma$ in $\Gamma$ enters the future and the past of $\hat{p}$. By Lemma $\mathrm{N}$, we see that this happens in a normal coordinate 
neighborhood of $p$, for fibers close enough to $\gamma$. But, in such a neighborhood, the boundaries of the past and the future of $\hat{p}$ are the lightcone of $\hat{p}$, so we may conclude that fibers close to $\gamma$ in $\Gamma$ intersect the past and future lightcones of $\hat{p}$. From topological considerations, we then see that every fiber near $\gamma=\pi^{-1}(p)$ intersects the lightcone of $\hat{p}$, and we are done.

The first lemma is a generalization of part of Proposition 7.27 of Penrose [20], and our proof follows its proof closely. Before stating the lemma, we recall the notions of null focal point and null coordinate system as set forth in [20].

Let $\gamma_{0}$ be a null geodesic orthogonal to a spacelike $(n-2)$-surface $\Sigma_{0}$ at a point $p$. A point $q \in \gamma_{0}, q \neq p$, is said to be a focal point to $\Sigma_{0}$ along $\gamma_{0}$ of order $k$ if and only if there exist $k$ nontrivial independent Jacobi fields along $\gamma_{0}$ which vanish at $q$ and which arise from a $k$-parameter variation of $\gamma_{0}$ through affinely parametrized null geodesics orthogonal to $\Sigma_{0}$.

For the construction of the null coordinate system, let $\Omega_{0}$ be the null hypersurface (near $\Sigma_{0}$ ) generated by the family $(\gamma)$ containing $\gamma_{0}$ of null geodesics orthogonal to $\Sigma_{0}$. Varying $\Sigma_{0}$ smoothly in some one-parameter family $(\Sigma)$ such that the direction of variation is not tangent to $\Omega_{0}$, we obtain a one-parameter family of null hypersurfaces $(\Omega)$. The null generators of $(\Omega)$ form a congruence extending $(\gamma)$. Let $T$ be the null vector field tangent to this congruence; then $T=-\nabla u$, where $u$ is the variation parameter whose level sets are the hypersurfaces $(\Omega)$. The vector field $T$ is geodesic $\left(\nabla_{T} T=0\right)$ and rotation free. Let $v$ be the affine parameter on $\gamma$ with $\partial / \partial v=T$ and $v=0, u=$ const. on $(\Sigma)$. Choose the remaining $(n-2)$ coordinates $x_{i}$ so that each geodesic $\gamma$ is given by $u, x_{1}, \ldots, x_{n-2}=$ const. Then the metric may be written as

$$
d s^{2}=2 d u\left(d v+\frac{1}{2} a d u+b_{\lambda} d x^{\lambda}\right)+r_{\lambda \mu} d x^{\lambda} d x^{\mu}, \quad \lambda, \mu=1, \ldots,(n-2) .
$$

This coordinate system is valid near $\gamma_{0}$ until a focal point to $\Sigma_{0}$ is reached [20, Proposition 7.26]. The surfaces $(\Sigma)$ may be allowed to degenerate to points. In this case the focal points of $\Sigma_{0}=\{p\}$ are called conjugate points to $p$ along $\gamma_{0}$.

LEMMA P. Let $X^{n}$ be an n-dimensional Lorentz manifold of signature $(-,+$, $\ldots,+)$; let $p, q \in X$. Let $\gamma$ be a future-null geodesic from $p$ to $q$.

(a) If the first internal point of $\gamma$ which is conjugate to $p$ is conjugate of kth order, $1 \leq k \leq n-2$, then there is a $k$-parameter variation of $\gamma$ through timelike curves from $p$ to $q$.

(b) Let $\Sigma$ be an $(n-2)$-surface which is spacelike and contains $p$, such that $\gamma$ is orthogonal to $\Sigma$ at $p$ and there is a focal point of $\Sigma$ along $\gamma$ between $p$ and $q$. Assume that the first such focal point is of $k$ th order, $1 \leq k \leq n-2$. Then there is a $k$-parameter variation of $\gamma$ through timelike curves from $\Sigma$ to $q$. If $k=n-2$, every point of $\Sigma$ in a neighborhood of $p$ is in the past of $q$.

PROOF. Let $r$ be the first conjugate point to $p$, or focal point to $\Sigma$, beyond $p$ along $\gamma$; by assumption, it is of $k$ th order. By Proposition 7.26 [20], there is a null coordinate system $\mathfrak{S}$ valid in some neighborhood of the portion of $\gamma$ between $p$ and $r$, and valid also at $p$ in case (b).

Let $T=\gamma$. We may choose the $u$-coordinate of $\mathfrak{S}$ so that $\langle\partial / \partial u, \partial / \partial u\rangle<0$

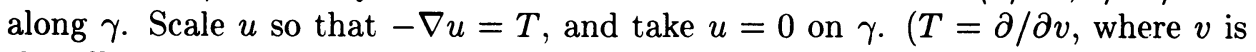
the affine parameter on $\gamma$.) 
Choose a point $w$ on $\gamma$ to the future of $r$, close enough to $r$ so that $r w$ contains no pair of conjugate points. Then, if $r^{\prime}$ precedes $r$ on $\gamma$ and is close enough to $r$, the point $r^{\prime}$ will not be conjugate to $w$ either. Thus the segment $r^{\prime} w$ of $\gamma$ is covered (except at $w$ ) by another null coordinate system $\mathfrak{S}$. Choose $\hat{\mathfrak{S}}$ so that $\hat{T}=\partial / \partial \hat{v}=\partial / \partial v=T$ along $\gamma$.

Since $r$ is a $k$ th-order conjugate point of $p$ (or focal point of $\Sigma$ ) along $\gamma$, there are $k$ independent nontrivial Jacobi fields $X_{1}, \ldots, X_{k}$ on $\gamma$ which vanish at $r$, and which arise from a $k$-parameter subfamily containing $\gamma$ of the congruence $(\gamma)$ of null lines of $\mathfrak{S}$ continuous with $\gamma$. We have $D X_{i} \neq 0, X_{i}=0$ at $r$. So, the $X_{i}$ have the form $X_{i}=\left(v_{0}-v\right) Y_{i}$, where $v_{0}$ is the value of $v$ at $r$. The $Y_{i}$ are smooth vector fields defined along $\gamma$ which are orthogonal to $\gamma$ (but not tangent to $\gamma$ ), and nonvanishing at $r$, so the $Y_{i}$ are spacelike at $r\left(\left.\left\langle Y_{i}, Y_{i}\right\rangle\right|_{r}>0\right)$. We may assume that the $Y_{i}$ are mutually orthogonal at $r^{\prime}\left(\left.\left\langle Y_{i}, Y_{j}\right\rangle\right|_{r^{\prime}}=0\right.$ if $\left.i \neq j\right)$.

Now

$$
\begin{aligned}
\left(Y_{i}\right)^{a}\left(Y_{j}\right)^{b} \nabla_{a} \nabla_{b} u & =-\left(Y_{i}\right)^{a}\left(Y_{j}\right)^{b} \nabla_{a} T_{b}=-\left\langle\nabla_{Y_{i}}, T, Y_{j}\right\rangle \\
& =-\left(v_{0}-v\right)^{-1}\left\langle\nabla_{X_{i}} T, Y_{j}\right\rangle=-\left(v_{0}-v\right)^{-1}\left\langle\nabla_{T}\left(v_{0}-v\right) Y_{i}, Y_{j}\right\rangle \\
& =\left(v_{0}-v\right)^{-1}\left\langle Y_{i}, Y_{j}\right\rangle-\left\langle\nabla_{T} Y_{i}, Y_{j}\right\rangle
\end{aligned}
$$

near $r$, this quantity being large and positive just to the past of $r$ on $\gamma$ if $i=j$. Thus, if the point $r^{\prime}$ is sufficiently close to $r$ and just before $r$ on $\gamma$, we have

$$
\left(Y_{i}\right)^{a}\left(Y_{i}\right)^{b} \nabla_{a} \nabla_{b}(u-\hat{u})>0 \quad \text { (formula P1) }
$$

at $r^{\prime}$, since $\nabla_{a} \nabla_{b} u$ is continuous at $r$. In fact, the matrix

$$
B_{i j}=\left.\left(Y_{i}\right)^{a}\left(Y_{j}\right)^{b} \nabla_{a} \nabla_{b}(u-\hat{u})\right|_{r^{\prime}}
$$

is positive definite.

Now set $U=\partial / \partial u$ at $r^{\prime}$, and consider $\exp _{r^{\prime}}$ applied to the $(k+1)$-plane $\Pi$ spanned by $U, Y_{1}, \ldots, Y_{k}$. Write the general element of $\Pi$ as $x U+y_{1} Y_{1}+\cdots+y_{k} Y_{k}$, and consider $u$ and $\hat{u}$ as functions of the coordinates $\left(x, y_{1}, \ldots, y_{k}\right)$. We have $u=\hat{u}=0$ at the origin $r^{\prime}=(0,0, \ldots, 0)$, and also $\partial u / \partial y_{i}=\partial \hat{u} / \partial y_{i}=0$ at $r^{\prime}$ (since $d u\left(Y_{i}\right)=-\left\langle Y_{i}, T\right\rangle=0$, and similarly for $\hat{u}$ ). Also $\partial u / \partial x=\partial \hat{u} / \partial x=1$ at $r^{\prime}$ (since $U(u)=-\langle U, T\rangle=1=-\langle U, \hat{T}\rangle=U(\hat{u})$ ). Finally, formula (P1) states that $A_{i j}-\hat{A}_{i j}$ is positive definite, where $A_{i j}=\partial^{2} u /\left.\partial_{y_{i}} \partial_{y_{j}}\right|_{r^{\prime}}$.

Consider the hypersurface

$$
4 x+\sum_{i, j=1}^{k}\left(A_{i j}+\hat{A}_{i j}\right) y_{i} y_{j}=0
$$

(hypersurface (P2)) in П. Taylor's theorem gives

$$
\begin{aligned}
& u=\frac{1}{4} \sum_{i, j=1}^{k}\left(A_{i j}-\hat{A}_{i j}\right) y_{i} y_{j}+o\left(y_{i} y_{j}\right), \quad 1 \leq j \leq k, \\
& \hat{u}=\frac{1}{4} \sum_{i=1}^{k}\left(\hat{A}_{i j}-A_{i j}\right) y_{i} y_{j}+o\left(y_{i} y_{j}\right),
\end{aligned}
$$

so for small enough $\left\|\left(y_{1}, \ldots, y_{k}\right)\right\|>0$, we have $\hat{u}<0<u$. Thus, $\exp _{r^{\prime}}$ applied to this value of $\left(x, y_{1}, \ldots, y_{k}\right)$ gives us a point $r^{\prime \prime}$ for which $\hat{u}>0$, so $r^{\prime \prime} \ll w$, and 
for which $u>0$, so (in case (a)) $p \ll r^{\prime \prime}$. That is, there exists a broken causal curve from $p$ to $w$ passing through $r^{\prime \prime}$. In fact, for each value of $\left(x, y_{1}, \ldots, y_{k}\right)$ on the hypersurface $(\mathrm{P} 2)$, such that $\left\|\left(y_{1}, \ldots, y_{k}\right)\right\|$ is small enough, there exists such a curve passing through $r^{\prime \prime}\left(y_{1}, \ldots, y_{k}\right)=\exp _{r^{\prime}}\left(x, y_{1}, \ldots, y_{k}\right)$. Therefore, there exists a $k$-parameter variation of $\gamma$ through broken causal curves from $p$ to $q$ which are not null geodesics. By Proposition 4.5.10 of Hawking and Ellis [12], such curves can be varied to give timelike curves joining $p$ and $q$, so case (a) is done.

To complete the proof of part (b), recall that $r^{\prime}$ is covered by the null coordinate system $\mathfrak{S}$; for $\left\|\left(y_{1}, \ldots, y_{k}\right)\right\|$ small enough, $r^{\prime \prime}\left(y_{1}, \ldots, y_{k}\right)$ will be covered by $\mathfrak{S}$ also. Let $\left(u, v, w_{1}, \ldots, w_{k}, z_{k+1}, \ldots, z_{n-2}\right)$ be the $n$-tuple representing the coordinates of $r^{\prime \prime}$ in $\mathfrak{S}$. We have shown above that $u\left(r^{\prime \prime}\right)$ and $v\left(r^{\prime \prime}\right)$ are positive for each $r^{\prime \prime}$. By construction, $u=v=0$ on $\Sigma$. Therefore,

$$
r^{\prime \prime}=\left(u, v, w_{1}, \ldots, w_{k}, z_{k+1}, \ldots, z_{n-1}\right) \gg\left(0,0, w_{1}, \ldots, w_{k}, z_{k+1}, \ldots, z_{n-2}\right) \in \Sigma .
$$

So there exists a broken causal curve from the point $\left(0,0, w_{1}, \ldots, w_{k}, z_{k+1}, \ldots, z_{n-2}\right)$ of $\Sigma$ to $q$ passing through $r^{\prime \prime}\left(y_{1}, \ldots, y_{k}\right)=\left(u, v, w_{1}, \ldots, w_{k}, z_{k+1}, \ldots, z_{n-2}\right)$. By Proposition 4.5 .10 of [12], this can be varied to give a timelike curve. Thus there is a $k$-parameter variation of $\gamma$ through timelike curves from $\Sigma$ to $q$. If $k=n-2$, each point of $\Sigma$ in a neighborhood of $p$ can be joined to $q$ by a timelike curve.

For the second lemma, recall that a neighborhood $U$ of a point $p$ in a pseudoRiemannian manifold $X,\langle$,$\rangle is called a normal coordinate neighborhood of p$ if there is a neighborhood $\tilde{U}$ of $O_{p}$ in $T_{p} M$ on which the exponential map $\exp _{p}$ is a diffeomorphism onto $U$. A normal neighborhood $U$ of $p$ is geodesically star-shaped with respect to $p$ (but not necessarily geodesically convex).

We derive the second lemma as a consequence of the following proposition.

Proposition. Let $X$ and $Y$ be locally compact Hausdorff spaces. Let $f: X \rightarrow$ $Y$ be a local homeomorphism, and let $A$ be a compact subset of $X$ such that $\left.f\right|_{A}$ is injective. Then there is an open subset $U$ of $X$ containing $A$ such that $\left.f\right|_{U}$ is a homeomorphism.

ProOF. If no such open set $U$ containing $A$ exists, there must be sequences of points $\left\{x_{\alpha}\right\},\left\{y_{\alpha}\right\}$ of $X$ arbitrarily close to $A$ such that $f\left(x_{\alpha}\right)=f\left(y_{\alpha}\right)$. Let $V$ be an open subset of $X$ containing $A$ such that the closure $\bar{V}$ of $V$ is compact. Within $\bar{V}$, choose convergent subsequences $x_{i} \rightarrow x$ and $y_{i} \rightarrow y$ with $x, y \in A$. Since $f$ is continuous, $f(x)=f(y)$.

If $x \neq y$, then $f$ is not injective on $A$. But if $x=y$, then $f$ is not one-to-one on any neighborhood of $x$, and thus $f$ cannot be a local homeomorphism.

Thus no such sequences can exist, and there is some neighborhood $U$ of $A$ in $X$ such that $\left.f\right|_{U}$ is a homeomorphism.

COROLlARY (LEMMA N). Let $\gamma([a, b]), 0 \in(a, b)$, be a segment of a geodesic in a pseudo-Riemannian manifold $Y$, such that $\gamma([a, b])$ has no self-intersections and contains no point conjugate to $\gamma(0)$. Then there is a neighborhood $U$ of $\gamma([a, b])$ which is a normal neighborhood of $\gamma(0)$.

PROOF. Apply the Proposition with $X=T_{\gamma(0)} Y, A=\exp _{\gamma(0)}^{-1}(\gamma([a, b]))$, and $f=\exp _{\gamma(0)}$, to obtain a neighborhood $\tilde{U}$ of $\gamma([a, b])$ on which $\exp _{\gamma(0)}$ is invertible. Then choose a neighborhood of $A=\exp _{\gamma(0)}^{-1}(\gamma([a, b]))$ contained in $\exp _{(0)}^{-1}(\tilde{U})$ which 
is star-shaped with respect to $0 \in T_{\gamma(0)} Y$; this is possible because $A$ is star-shaped with respect to 0 . The image of this star-shaped neighborhood is the required normal neighborhood $U$ of $\gamma(0)$ containing $\gamma([a, b])$.

Proof of TheOREM. Fix a point $p \in M$, and a point $\hat{p} \in \pi^{-1}(p) \subset L$. Let $\gamma(t)$ be a parametrization of $\pi^{-1}(p)$ such that $\dot{\gamma}=K$ and $\gamma(0)=\hat{p}$; then $\gamma(t)$ is an affinely parametrized null geodesic of $L$.

We know that sectional curvatures of all vertical null planes $K \wedge X$ in $T_{\gamma(t)} L$ are equal and positive, for all $t$. An examination of Jacobi's equation shows that all Jacobi fields $J(t)$ along $\gamma(t)$ with $J^{\prime}(0)=0$ and $J(0)=X$, where $X$ is some spacelike vector perpendicular to $K$, vanish simultaneously at a point $\gamma(\pi / 2 \sqrt{\kappa})$ to the future of $\gamma(0)$ on $\gamma$, and at a point $\gamma(-\pi / 2 \sqrt{\kappa})$ to the past of $\gamma(0)$ on $\gamma$, where $\kappa=\kappa_{K}(K \wedge X)$.

Let $\Sigma^{2 n}$ be a portion of a wavefront of the lightcone of $\gamma(\pi / 2 \sqrt{\kappa})$ at $\hat{p}=\gamma(0)$. Then $\Sigma$ is a spacelike surface of dimension $2 n$, perpendicular to $\gamma$ at $\hat{p}$, and having past and future null focal points of order $2 n$ along $\gamma$ at affine distances $-\pi / 2 \sqrt{\kappa}$ and $+\pi / 2 \sqrt{\kappa}$ respectively.

Next, let $\Gamma^{2 n+1}$ be the vertical hypersurface in $L$ consisting of those fibers of $\pi$ which pass through $\Sigma^{2 n}$, i.e., let $\Gamma=\pi^{-1}(\pi(\Sigma))$. Let $\gamma(a)$ be a point of $\gamma$ to the past of $\hat{p}=\gamma(0)$, which lies between the first past-conjugate-point of $\hat{p}$ along $\gamma$ and the first past-focal-point of $\Sigma$ along $\gamma$. Choose also a point $\gamma(b)$ between the first future focal and conjugate points of $\hat{p}$ on $\gamma$. Since the vertical null vector field $K$ on $L$ is a Killing field, the hypersurface $\Gamma$ is foliated by the past and future translates of $\Sigma$ under the action of $K$. Let $\Sigma_{a}$ be the past-translate of $\Sigma$ on which the point $\gamma(a)$ lies; let $\Sigma_{b}$ be the future-translate of $\Sigma$ containing $\gamma(b)$. By Lemma P, every point of $\Sigma_{a}$ close enough to $\gamma(a)$ lies in the past of $\hat{p}$ (denoted $J^{-}(\hat{p})$ ), and every point of $\Sigma_{b}$ near $\gamma(b)$ lies in the future of $\hat{p}$ (denoted $J^{+}(\hat{p})$ ). In terms of $\Gamma$, this means that every fiber of $\pi$ in $\Gamma$ near $\gamma$ enters the future of $\hat{p}$ and the past of $\hat{p}$ before reaching $\Sigma_{b}$ and $\Sigma_{a}$, respectively.

By Lemma $\mathrm{N}$, there is a neighborhood $U$ of $\gamma([a, b])$ which is a normal coordinate neighborhood of $\hat{p}=\gamma(0)$. In such a neighborhood, the proof of Proposition 4.5.1 (Hawking and Ellis [12]) shows that the boundary $\partial J^{+}(p)$ of the future of $\hat{p}$ is the future lightcone of $\hat{p}$ (which we will denote $L C^{+}(\hat{p})$ ); also $\partial J^{-}(\hat{p})=L C^{-}(\hat{p})$. We now see that those fibers of $\pi$ which enter $J^{ \pm}(\hat{p})$ before leaving $U$ must intersect $L C^{ \pm}(\hat{p})$. Since $U$ is an open neighborhood of $\gamma([a, b])$, we may say that every fiber of $\pi$ close enough to $\gamma$ in $\Gamma$ intersects both $L C^{+}(\hat{p})$ and $L C^{-}(\hat{p})$. Now, the lightcone of $\hat{p}$ is ruled by the null geodesics which pass through $p$. By the "conservation lemma" (also called the "constant-of-motion lemma"), any geodesic of $L$ has a constant inner product with the Killing field $K$ which is tangent to the fibers of $\pi$. Thus the lightcone of $p$ is transverse to the fibers of $\pi$, except along $\gamma=\pi^{-1}(p)$. That is, $\pi$ restricted to $L C-\gamma$ is a local homeomorphism.

In fact, $\pi$ restricted to $\left(L C^{+}-\gamma\right) \cap U$ is a homeomorphism. If any two points $q_{1}$ and $q_{2}$ of $\left(L C^{+}-\gamma\right) \cap U$ lay on the same fiber, then the one (say $\left.q_{2}\right)$ to the future could be reached from $p$ by a broken null geodesic consisting of the light ray from $\hat{p}$ to $q_{1}$, followed by the section of the fiber (which is also a null geodesic) from $q_{1}$ to $q_{2}$. This curve is not a smooth null geodesic, since the first segment is nonvertical and the second is vertical, so by Proposition 4.5.10 of Hawking and Ellis $[\mathbf{1 2}], q_{2}$ can be reached from $\hat{p}$ by some timelike curve. Thus curve can be made to 
lie pointwise close to the broken null geodesic from $\hat{p}$ to $q_{2}$, and thus to lie in $U$. But then $q_{2}$ could not have been a point of $L C^{+}(\hat{p})$, since $L C^{+}(\hat{p}) \subset \partial J^{+}(\hat{p})$ in $U$. So no two points of $\left(L C^{+}-\gamma\right) \cap U$ can lie on the same fiber, and $\left.\pi\right|_{\left(L C^{+}-\gamma\right) \cap U}$ is one-to-one. Thus $\left.\pi\right|_{\left(L C^{+-}-\gamma\right) \cap U}$ is homeomorphism. Similarly, $\left.\pi\right|_{\left(L C^{-}-\gamma\right) \cap U}$ is a homeomorphism.

Finally, we must show that $\pi$ maps a suitable subset of the lightcone of $\hat{p}$ onto an open neighborhood $V$ of $p$ in $M$. To this end, define a subset $\mathcal{L}^{+}$of $\left(L C^{+}-\gamma\right) \cap U$ as follows: include in $\mathcal{L}^{+}$the open segment of each future-null geodesic through $\hat{p}$, from $\hat{p}$ to the point where the geodesic either (1) leaves $U$, or (2) intersects $\Gamma$, whichever comes first. In case (2), include also the intersection point. Define a set $\mathcal{L}^{-}$similarly.

From $\mathcal{L}^{+}$and $\mathcal{L}^{-}$we shall construct a new set $S$. Choose $t^{+}, t^{-} \in \mathbf{R}, t^{-}<0<$ $t^{+}$, so that $\gamma\left(t^{-}\right)$is to the past of the first past-conjugate-point of $\gamma(0)$ along $\gamma$, and so that $\gamma\left(t^{+}\right)$is to the future of the first future-conjugate-point of $\gamma(0)$ along $\gamma$. Let $S$ be the union of (1) a copy of $\mathcal{L}^{+}$, past-translated an affine distance $t^{-}$ under the action of $K,(2)$ a copy of $\mathcal{L}^{-}$, future-translated an affine distance $t^{+},(3)$ those segments of fibers in $\Gamma$ intersecting both the translates of $\mathcal{L}^{+}$and $\mathcal{L}^{-}$which lie between the intersection points, and (4) the closed segment of $\gamma$ from $\gamma\left(t^{-}\right)$to $\gamma\left(t^{+}\right) . S$ is homeomorphic to an open $(2 n+1)$-ball; $\hat{p}=\gamma(0)$ is in the interior of $S$.

Now define an equivalence relation $R$ on $S$ by declaring two points $\alpha$ and $\beta$ of $S$ to be $R$-equivalent if $\pi(\alpha)=\pi(\beta)$. Then the set $B=S / R$ is still homeomorphic to an open $(2 n+1)$-ball, and the equivalence class $[\hat{p}]$ of $\hat{p}$ is in the interior of $B$.

The projection map $\pi: L \rightarrow M$ induces a map $\tilde{\pi}: B \rightarrow M$ which, by the above discussion, is manifestly one-to-one and continuous. By the invariance of domain theorem [24], the set $V=\tilde{\pi}(B)$ is an open $(2 n+1)$-ball in $M$, and $\pi(\hat{p})=p$ is in the interior of $V$.

Since every point of the sets $\mathcal{L}^{+}$and $\mathcal{L}^{-}$can be reached from $\hat{p}$ by a null geodesic, and since the image of $\mathcal{L}^{+} \cup \mathcal{L}^{-}$under $\pi$ is the same as the image of $B$ under $\tilde{\pi}$ (that is, $V$ ) by construction, we have shown that a point $p$ of $M$ admits a neighborhood $V$ such that every point $q$ of $V, q \neq p$, can be reached from $p$ by the projection of a null geodesic of $L$. That is, $p$ and $q$ can be connected by an $L$-pseudochain.

V. Example of a nonstandard pseudochain system on the Heisenberg group. We now turn to the study of an example of a three-dimensional CR manifold with a system of pseudochains which are not chains. We have noted that Fefferman metrics satisfy $K\rfloor C=0$, where $C$ is the Weyl tensor; thus a fourdimensional Fefferman metric must be of type $N$ or 0 in the Petrov classification (see Kramer et al. [16]), and Lorentz manifolds of other Petrov types cannot be Fefferman. We shall study such a metric with a rotating null Killing field, identify its quotient CR manifold, and show that the pseudochains of the induced system differ qualitatively from the standard chains on this CR manifold.

The Lorentz manifold we wish to study is the Gödel universe [9], which we label $G^{4}$. This spacetime is homeomorphic to $\mathbf{R}^{4}$; and carries the Lorentz metric

$$
d s^{2}=d \hat{x}^{2}+d \hat{z}^{2}+\frac{1}{2} e^{2 \hat{x}} d \hat{y}^{2}-\left(d \hat{t}+e^{\hat{x}} d \hat{y}\right)^{2} .
$$

It is a solution of the Einstein equations (with cosmological constant) for a pressurefree perfect fluid, and it is of Petrov type $D[\mathbf{1 6}]$. Let us write $\hat{T}=\partial / \partial \hat{t}, \hat{X}=\partial / \partial \hat{x}$, $\hat{Y}=\partial / \partial \hat{y}, \hat{Z}=\partial / \partial \hat{z}$. The two null congruences $\hat{T}+\hat{Z}$ and $\hat{T}-\hat{Z}$ are in fact 
commuting null Killing fields with nonvanishing rotation. Let $K_{1}=\hat{T}+\hat{Z}$ and $K_{2}=\hat{T}-\hat{Z}$. We shall consider the quotient CR manifold $M^{3}$ of $G^{4}$ by the null geodesics tangent to the Killing field $K=\sqrt{2} K_{1}$.

The space $M^{3}$ can be realized in $G^{4}$ as the subspace $\hat{z}=-\hat{t}$; we give it the coordinates $(\tau, x, y)$, where $\tau=\hat{t}-\hat{z}, x=\hat{x}$, and $y=\hat{y}$. The holomorphic tangent plane field on $M$ is found by projecting $K^{\perp}$ to $T M$; it is spanned by $X=\pi_{*}(\hat{X})$ and $Y=\pi_{*}\left(\hat{T}-e^{-\hat{x}} \hat{Y}\right)$. $\left(\hat{X}\right.$ and $\hat{T}-e^{-\hat{x}} Y$ are orthonormal spacelike vector fields of $G$.) The CR structure tensor $J$ on $M$ is given by $J=\pi_{*}(\nabla K)$; we see that $J(X)=Y$ and $J(Y)=-X$.

The Gödel universe is a completely homogeneous space-time. It has the five symmetries [9]:

(1) $\hat{t} \rightarrow \hat{t}+b$

(2) $\hat{y} \rightarrow \hat{y}+b$

(3) $\hat{z} \rightarrow \hat{z}+b$,

(4) $\hat{x} \rightarrow \hat{x}+b, \hat{y} \rightarrow e^{-b} \hat{y}$,

(5) a rotation (best described in other coordinates).

These induce the following four symmetries of $M$ :

(1) $\tau \rightarrow \tau+b$

(2) $y \rightarrow y+b$

(3) $x \rightarrow x+b, y \rightarrow e^{-b} y$,

(4) rotation.

Thus $M$ is a homogeneous CR-manifold. Symmetries (1)-(3) leave invariant the vector fields

(1) $T=\pi_{*}\left(K_{2}\right)$,

(2) $X=\pi_{*}(\hat{X})$

(3) $W=\pi_{*}\left(\sqrt{2} e^{-x} \hat{Y}\right)=\sqrt{2} T=Y$.

These vector fields form a Lie algebra with the product relations

$$
[T, X]=[T, W]=0 ; \quad[W, X]=W .
$$

Thus we see that $M$ is a Lie group isomorphic to $\mathbf{R} \times P^{2}$, where $P^{2}$ is the noncommutative half-plane. The identity element is the point $(\tau, x, y)=(0,0,0)$.

The homogeneous 3-dimensional CR manifolds have been classified by Cartan [4]. The space $M$ we have been considering is called $\Sigma_{B}$ in Cartan's classification (see no. 37 of $[4]$ ), and it is locally equivalent to the Heisenberg group $H^{3}=$ $\left\{(U, V) \mathbf{C}^{2} \mid U-\bar{U}-i V \bar{V}=0\right\}$. In fact, $M$ may be thought of as the universal cover of the Heisenberg group with a chain through infinity, $\{(u, v) \mid V=0\}$, removed. The map from $M^{3}$ in three real coordinates $(\tau, x, y)$ to $H^{3}$ given as a subset of $\mathbf{C}^{2}$ in two complex coordinates $(U, V)$ is given by

$$
U=\frac{y+i e^{-x}}{2}, \quad V=e \frac{-x+i \tau}{2} .
$$

(Our coordinates $(\tau, x, y)$ on $M^{3}$ correspond to Cartan's coordinates $(a, b, c)$ on $\Sigma_{B}$ as follows: $\tau=b, x=-c, y=a$.) The point $(0,0,0)$ of $M$ is sent to the point $(i / 2,1)$ of $H$ in $\mathbf{C}^{2}$.

The null geodesics of $G^{4}$ project to a system of pseudochains on $M$. We have shown that two sufficiently nearby points of $M$ are connected by a curve of this 
system. However, these pseudochains differ markedly in their local and global behavior from the standard chains. We shall map the $G^{4}$-pseudochains of $M$ to the Heisenberg group, where the standard chains are well known, in order to make the comparison. There we shall find that $G^{4}$-pseudochains are never closed in sufficiently small neighborhoods, and that they do not connect pairs of points globally. This, of course, is in striking contrast to the behavior of the standard chains of $H^{3}$, some of which are circles in any neighborhood, and which do connect any pair of points.

To show the difference in the global behavior of chains and $G$-pseudochains, consider the function $r$ on $G$ given by

$$
\cosh (2 r)=\frac{1+e^{2 \hat{x}}+\frac{1}{2} \hat{y}^{2} e^{2 \hat{x}}}{2 e^{\hat{x}}} .
$$

Chandrasekhar and Wright [5] computed the geodesics of the Gödel universe $G$, and they found that this function $r$ is bounded above by the value $r_{0}=\ln (1+\sqrt{2})$ on the null geodesics which pass through $(\hat{t}, \hat{x}, \hat{y}, \hat{z})=(0,0,0,0)$. Projecting $r$ down to a function $\tilde{r}$ on $M$, we see that $\tilde{r}$ is bounded by $\ln (1+\sqrt{2})$ on the $G$-pseudochains passing through $(\tau, x, y)=(0,0,0)$. Thus

$$
3 \geq \cosh (2 r)=\frac{1+e^{2 x}+\frac{1}{2} y^{2} e^{2 x}}{2 e^{x}} .
$$

Now passing from $M^{3}$ to $H^{3}$ via the correspondence given above, we find that

$$
3 \geq \frac{1+|V|^{-4}+2 \operatorname{Re}^{2}(U)|V|^{-4}}{2|V|^{-2}}
$$

on the $G$-pseudochains passing through the point $(i / 2,1)$ of $H$. Since $2 \operatorname{Im}(U)=$ $|V|^{2}$ on $H$, we may rewrite this inequality as

$$
3 \geq \frac{1+\frac{1}{4} \operatorname{Im}^{-2}(U)+\frac{1}{2} \operatorname{Re}^{2}(U) \operatorname{Im}^{-2}(U)}{\operatorname{Im}^{-1}(U)} .
$$

Simplifying, we have

$$
-2 \operatorname{Re}^{2}(U) \geq 4 \operatorname{Im}^{2}(U)-12 \operatorname{Im}(U)+1 .
$$

In order for this inequality to have solutions, it is necessary that $-2 \leq \operatorname{Re}(U) \leq 2$. Even if $\operatorname{Re}(U)=0$, which allows $\operatorname{Im}(U)$ to take on the widest possible range of values, we find that

$$
(3-2 \sqrt{2}) / 2 \leq \operatorname{Im}(U) \leq(3+2 \sqrt{2}) / 2 .
$$

Using the defining equation $\operatorname{Im}(U)=\frac{1}{2}|V|^{2}$ of $H$, we see that the coordinates $(U, V)$ must remain in a compact subset of $\mathbf{C}^{2}$ on the pseudochains passing through $(i / 2,1)$, and so these pseudochains remain in a compact subset of $H$. Thus most points of $H$ cannot be joined to $(i / 2,1)$ by a $G$-pseudochain. The standard chains of $\mathrm{H}^{3}$ join any pair of points (even making allowances for the absence of points on the line $V=0)$, and so the standard chains through $(i / 2,1)$ do not remain in any compact set.

The pseudochains of this system also differ locally from the standard chains in that they are not closed in small neighborhoods. To see this, consider the func- 
tion $f$ given by restricting the $z$-coordinate of the Gödel universe to any affinely parametrized null geodesic. Since $\partial / \partial z$ is parallel, $f$ is a linear function of the affine parameter of the geodesic, and therefore it is strictly monotone and unbounded above and below. Since $\partial / \partial z$ is also nonvertical, $f$ projects to a function $F$ on pseudochains with the same properties; $F$ is the $\tau$-coordinate of a point of a pseudochain in the coordinates $(\tau, x, y)$ on $\Sigma_{B}$ discussed above. Mapping to the Heisenberg group, we see that $\operatorname{Arg}(V)$ is always decreasing on every pseudochain. Thus, no pseudochain of this system in a sufficiently small neighborhood of $H-\{V=0\}$ can be closed.

ACKNOWLEDGMENTS. This paper is based on the author's doctoral thesis, written under the direction of C. Denson Hill on a topic suggested by Claude R. LeBrun. The author would like to thank Professors Hill and LeBrun for their help and encouragement.

I would also like to thank the referee for suggestions which simplified the proof of a proposition.

\section{REFERENCES}

1. R. Bott and L. W. Tu, Differential forms in algebraic topology, Springer-Verlag, New York, 1982.

2. D. Burns and S. Shnider, Real hypersurfaces in complex manifolds, Several Complex Variables, Proc. Sympos. Pure Math., vol. 40, Amer. Math. Soc., Providence, R. I., 1977, pp. 141-168.

3. D. Burns, K. Diederich, and S. Shnider, Distinguished curves in pseudoconvex boundaries, Duke Math. J. 44 (1977), 407-431.

4. E. Cartan, Sur l'equivalence pseudo-conforme des hypersurfaces de l'espace de deux variables complexes. I, Ann. of Math. (2) 11 (1932), 17; II, Ann. Scuola Norm. Sup. Pisa 1 (1932), 333.

5. S. Chandrasekhar and J. P. Wright, The geodesics in Godel's universe, Proc. Nat. Acad. Sci. U.S.A. 47 (1961), 341.

6. S. S. Chern and J. K. Moser, Real hypersurfaces in complex manifolds, Acta Math. 133 (1974), 219271.

7. F. Farris, An intrinsic construction of Fefferman's CR metric, Pacific J. Math. 123 (1986), 33-45.

8. C. Fefferman, Monge-Ampere equations, the Bergman kernel, and the geometry of pseudoconvex domains, Ann. of Math. (2) 103 (1976), 395; Erratum, 104 (1976), 393.

9. K. Godel, An example of a new type of cosmological solution of Einstein's field equations of gravitation, Rev. Modern Phys. 21 (1949), 447-450.

10. C. R. Graham, On Sparling's characterization of Fefferman metrics, preprint.

11. S. Harris, A triangle comparison theorem for Lorentz manifolds, Indiana Univ. Math. J. 31 (1982), 289-308.

12. S. W. Hawking and G. F. R. Ellis, The large-scale structure of space-time, Cambridge Univ. Press, Cambridge, 1973.

13. M. W. Hirsch, Differential topology, Springer-Verlag, New York, 1976.

14. H. Jacobowitz, Chains in CR geometry, J. Differential Geom. 21 (1985), 163.

15. J. Lee, The Fefferman metric and pseudohermitian invariants, preprint.

16. D. Kramer, H. Stephani, E. Herlt, M. MacCallum, and E. Schmutzer (eds.), Exact solutions of Einstein's field equations, Cambridge Univ. Press, Cambridge, 1980.

17. S. Kobayashi and K. Nomizu, Foundations of differential geometry, I \& II, Interscience, New York, 1969.

18. L. Nirenberg, Lectures on partial differential equations, CBMS Regional Conf. Ser. Math., no. 17, Amer. Math. Soc., Providence, R. I., 1973.

19. __ On a question of Hans Lewy, Russian Math. Surveys 29 (1974), 241251. 
20. R. Penrose, Techniques of differential topology in relativity, CBMS Regional Conf. Ser. in Math., no. 7, Amer. Math. Soc., Providence, R. I., 1972.

21. H. Poincaré, Les fonctions analytiques de deux variables et la représentation conforme, Rend. Circ. Mat. Palermo (1907), 185.

22. A. Sparling, Twistor theory and the characterization of Fefferman's conformal structures, preprint.

23. S. Webster, Pseudo-Hermitian structures on a real hypersurface, J. Differential Geom. 13 (1978), 25-41.

24. E. Spanier, Algebraic topology, Springer-Verlag, New York, 1966.

Department of Mathematics, Rutgers UniVersity, NeW BRUNSWiCK, NeW JeRSEY 08903 